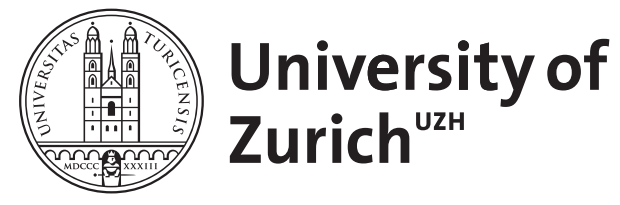

\title{
Estimates for Dirichlet eigenfunctions
}

van den Berg, M ; Bolthausen, E

\begin{abstract}
Estimates for the Dirichlet eigenfunctions near the boundary of an open, bounded set in euclidean space are obtained. It is assumed that the boundary satisfies a uniform capacitary density condition.
\end{abstract}

DOI: https://doi.org/10.1112/S0024610799007267

Posted at the Zurich Open Repository and Archive, University of Zurich ZORA URL: https://doi.org/10.5167/uzh-22158

Journal Article

Published Version

Originally published at:

van den Berg, M; Bolthausen, E (1999). Estimates for Dirichlet eigenfunctions. Journal- London Mathematical Society, 59(2):607-619.

DOI: https://doi.org/10.1112/S0024610799007267 


\title{
ESTIMATES FOR DIRICHLET EIGENFUNCTIONS
}

\author{
M. VAN DEN BERG AND E. BOLTHAUSEN
}

\begin{abstract}
Estimates for the Dirichlet eigenfunctions near the boundary of an open, bounded set in euclidean space are obtained. It is assumed that the boundary satisfies a uniform capacitary density condition.
\end{abstract}

\section{Introduction}

Let $D$ be an open, bounded set in euclidean space $\mathbb{R}^{m}(m=2,3, \ldots)$ with boundary $\partial D$. Let $-\Delta_{D}$ be the Dirichlet laplacian for $D$. The spectrum of $-\Delta_{D}$ is discrete and consists of eigenvalues $\lambda_{1} \leqslant \lambda_{2} \leqslant \ldots$ with a corresponding orthonormal set of eigenfunctions $\left\{\phi_{1}, \phi_{2}, \ldots\right\}$. The behaviour of the eigenfunctions near the boundary $\partial D$ of $D$ has been investigated by several authors under a variety of assumptions on the geometry of $D[\mathbf{1}-\mathbf{8}, \mathbf{1 1}-\mathbf{1 6}]$.

In this paper we obtain pointwise bounds on the eigenfunctions under the assumption that $\partial D$ satisfies a uniform capacitary density condition. Denote by $\operatorname{Cap}(A)$ the newtonian capacity of a compact set $A \subset \mathbb{R}^{m}(m=3,4, \ldots)$ or the logarithmic capacity of a compact set $A \subset \mathbb{R}^{2}$. For $x \in \mathbb{R}^{m}$ and $r>0$ we define

$$
B(x ; r)=\left\{y \in \mathbb{R}^{m}:|y-x| \leqslant r\right\},
$$

and for a non-empty set $G \subset \mathbb{R}^{m}$

$$
\operatorname{diam}(G)=\sup \left\{\left|x_{1}-x_{2}\right|: x_{1} \in G, x_{2} \in G\right\} .
$$

Definition 1.1. Let $D \subset \mathbb{R}^{m}(m=2,3, \ldots)$ be an open set with boundary $\partial D$. Then $\partial D$ satisfies an $\alpha$-uniform capacitary density condition if for some $\alpha \in(0,1]$

$$
\operatorname{Cap}((\partial D) \cap B(x ; r)) \geqslant \alpha \operatorname{Cap}(B(x ; r)), \quad x \in \partial D, \quad 0<r<\operatorname{diam}(D) .
$$

Condition (1.3) guarantees that all points of $\partial D$ are regular, and in particular that $\lim _{x \rightarrow x_{0}} \phi_{j}(x)=0$ for all $x_{0} \in \partial D$. Definition 1.1 has been introduced in [10] in a study of the partition function of the Dirichlet laplacian on open sets with a non-smooth or fractal boundary.

Let $d: D \longrightarrow(0, \infty)$ denote the distance function

$$
d(x)=\min \{|x-y|: y \in \partial D\},
$$

and let $R$ be the inradius of $D$, defined by

$$
R=\max _{x \in D} d(x) .
$$

The main results of this paper are the following.

Received 26 January 1996; revised 2 October 1996.

1991 Mathematics Subject Classification 35J25, 60J65.

Part of this research was funded by the British Council and the Swiss National Science Foundation. J. London Math. Soc. (2) 59 (1999) 607-619 
Theorem 1.2. Let $D$ be an open, bounded set in $\mathbb{R}^{2}$. Suppose $\partial D$ satisfies (1.3) for some $\alpha>0$. Then for $j=1,2, \ldots$ and all $x \in D$ such that $d(x)<\lambda_{j}^{-1 / 2}$

$$
\left|\phi_{j}(x)\right| \leqslant\left\{6 \lambda_{j} \log \left(2 / \alpha^{2 \pi}\right) \frac{-1}{\log \left(d(x) \lambda_{j}^{1 / 2}\right)}\right\}^{1 / 2} .
$$

Theorem 1.3. Let $D$ be an open, bounded set in $\mathbb{R}^{m}(m=3,4, \ldots)$. Suppose $\partial D$ satisfies (1.3) for some $\alpha>0$. Let $j=1,2, \ldots$ and suppose $x \in D$ satisfies

$$
d(x) \lambda_{j}^{1 / 2} \leqslant\left(\frac{\alpha^{6}}{2^{13}}\right)^{1+\gamma(m-1) /(m-2)} .
$$

Then

where

$$
\left|\phi_{j}(x)\right| \leqslant 2 \lambda_{j}^{m / 4}\left(d(x) \lambda_{j}^{1 / 2}\right)^{(1 / 2)((1 / \gamma)+(m-1) /(m-2))^{-1}}
$$

$$
\gamma=\frac{3^{-m-1} \alpha}{\log \left(2(2 / \alpha)^{1 /(m-2)}\right)} .
$$

The bounds in (1.6) and (1.8) are being complemented by the following wellknown estimate [11, Lemma 3.1].

THEOREM 1.4. Let D be an open, bounded set in $\mathbb{R}^{m}(m=2,3, \ldots)$. Then for $j=1,2, \ldots$ and $x \in D$

$$
\left|\phi_{j}(x)\right| \leqslant \lambda_{j}^{m / 4} \text {. }
$$

The bounds of Theorems 1.2 and 1.3 are in general not sharp. For example if $D$ is open, bounded and $\partial D$ is smooth then the eigenfunctions are comparable with $d(x)$. If $D$ is open, bounded and simply connected in $\mathbb{R}^{2}$ then it was shown by Bañuelos [3, Corollary 2.3b] that $\phi_{1}$ is comparable to the hyperbolic distance induced by the conformal map $F$ from the unit disc onto $D$. By Koebe's 1/4 theorem [17] one then obtains that

$$
\phi_{1}(x) \leqslant C d(x)^{1 / 2}
$$

for some constant $C$ depending on $D$. We will use the ideas of [3] to prove (in Section $5)$ the following refinement of (1.11).

THeOREM 1.5. Let $D$ be an open, simply connected set in $\mathbb{R}^{2}$ with volume $|D|$. Then for $j=1,2, \ldots$

$$
\left|\phi_{j}(x)\right| \leqslant 2^{9 / 2} \pi^{1 / 4} j|D|^{1 / 4} R^{-2} d(x)^{1 / 2} .
$$

The following example (see $[\mathbf{1 3}, 4.6 .7])$ shows that Theorem 1.5 is sharp.

EXAMPLE 1.6. Let $U \subset \mathbb{R}^{m}$ be the conical region in polar coordinates defined by

$$
U=\{(r, \omega): 0<r<1, \omega \in \Omega\},
$$

where $\Omega$ is an open subset of the unit sphere $S^{m-1}$. Then

$$
\varphi_{1}(r, \omega) \asymp r^{\beta(\Omega)},
$$

where $\beta(\Omega)$ is the positive solution of

$$
\beta(\beta+m-2)=\lambda_{1}(\Omega),
$$

and where $\lambda_{1}(\Omega)$ is the first eigenvalue of the Laplace-Beltrami operator on $\Omega$ with Dirichlet conditions of $\partial \Omega$. In particular if $m=2$ and

$$
\Omega_{0}=\left\{\omega \in S^{1}: 0<\omega<2 \pi\right\},
$$


then

and by (1.15)

$$
\lambda_{1}\left(\Omega_{0}\right)=\frac{1}{4}
$$

$$
\beta\left(\Omega_{0}\right)=\frac{1}{2},
$$

which shows that the exponent in (1.12) cannot be improved.

The main idea of the proofs of Theorems 1.2 and 1.3 goes back to Brossard and Carmona [10] who obtained estimates for the Dirichlet heat kernel $p_{D}(x, x ; t)$ for $x$ near $D$. We improve their lemma [10, Lemma 3.5] and its proof (see also [9]). In the proof of Theorem 1.3 we also require a refinement of Wiener's test [18].

Let $\left(B(s), s \geqslant 0 ; \mathbb{P}_{x}, x \in \mathbb{R}^{m}\right)$ be a brownian motion associated to $-\Delta+\partial / \partial t$. Let $T_{D}$ denote the first exit time of the brownian motion from $D$ :

$$
T_{D}=\inf \{s \geqslant 0: B(s) \in \partial D\} .
$$

For a compact set $K$ we also define the first entry time

Then

$$
\tau_{K}=\inf \{s>0: B(s) \in K\} .
$$

$$
\mathbb{P}_{x}\left[T_{D}>t\right]=\int_{D} p_{D}(x, y ; t) d y .
$$

By the eigenfunction expansion of the heat kernel and by the semigroup property we have

$$
e^{-t \lambda_{j}} \phi_{j}^{2}(x) \leqslant \sum_{j=1}^{\infty} e^{-t \lambda_{j}} \phi_{j}^{2}(x)=p_{D}(x, x ; t)=\int_{D} p_{D}^{2}(x, y ; t / 2) d y .
$$

Since the Dirichlet heat kernel is monotone in $D$

Hence

$$
p_{D}(x, y ; t / 2) \leqslant p_{\mathbb{R}^{m}}(x, y ; t / 2) \leqq(2 \pi t)^{-m / 2} .
$$

$$
e^{-t \lambda_{j}} \phi_{j}^{2}(x) \leqslant(2 \pi t)^{-m / 2} \int_{D} p_{D}(x, y ; t / 2) d y=(2 \pi t)^{-m / 2} \mathbb{P}_{x}\left[T_{D}>t / 2\right] .
$$

The choice

yields for $m=2,3, \ldots$

$$
t=2 \lambda_{j}^{-1}
$$

$$
\left|\phi_{j}(x)\right| \leqslant e(4 \pi)^{-m / 4} \lambda_{j}^{m / 4}\left(\mathbb{P}_{x}\left[T_{D}>\lambda_{j}^{-1}\right]\right)^{1 / 2} \leqslant \lambda_{j}^{m / 4}\left(\mathbb{P}_{x}\left[T_{D}>\lambda_{j}^{-1}\right]\right)^{1 / 2} .
$$

This proves Theorem 1.4 since $\mathbb{P}_{x}\left[T_{D}>\lambda_{j}^{-1}\right] \leqslant 1$.

In Sections 2 and 3 we obtain the upper bounds for $\mathbb{P}_{x}\left[T_{D}>\lambda_{j}^{-1}\right]$ in the cases $m=2$ and $m=3,4, \ldots$ respectively. In the proof of Theorem 1.3 we use the following modification of Wiener's test. See also [20, Theorem 4.7, p. 73] for related refinements of Wiener's test.

Theorem 1.7. Let $D$ be an open, bounded set in $\mathbb{R}^{m}(m=3,4, \ldots)$. Suppose $\partial D$ satisfies (1.3) for some $\alpha>0$. If $x \in D$ satisfies $d(x) \leqslant\left(\alpha^{6} / 2^{13}\right) \operatorname{diam}(D)$, then for any

one has

$$
a \in\left[\frac{2^{13}}{\alpha^{6}}, \operatorname{diam}(D) / d(x)\right]
$$

$$
\mathbb{P}_{x}\left[\tau_{(\partial D) \cap B(x ; a d(x))}<\infty\right] \geqslant 1-2 a^{-\gamma},
$$

where $\gamma$ is given by (1.9). 
The proof of Theorem 1.7 will be deferred to Section 4 .

\section{Proof of Theorem 1.2}

Let $m=2$ and define the Green function

$$
g(x, y)=-(2 \pi)^{-1} \log |x-y| .
$$

The equilibrium measure on a compact set $K \subset \mathbb{R}^{2}$ is the unique probability measure $\mu_{K}$ concentrated on $K$ for which

$$
u_{K}(x)=\int_{K} g(x, y) \mu_{K}(d y)
$$

is constant on the regular points of $K$. The function $u_{K}$ is the equilibrium potential of $K$ and its value $R(K)$ on the regular points of $K$ is the Robin constant. The logarithmic capacity is defined by

Then

$$
\operatorname{Cap}(K)=e^{-R(K)} .
$$

$$
\operatorname{Cap}(K)=\exp -\left\{\inf _{\mu \in P(K)} \int_{K} \int_{K} g(x, y) \mu(d x) \mu(d y)\right\},
$$

where $P(K)$ is the set of all probability measures supported by $K$. Moreover

$$
\operatorname{Cap}(B(x ; r))=r^{1 /(2 \pi)}
$$

[18, Chapter 3, Proposition 4.11]. Define

Let $a>4$. Then

$$
B^{\circ}(x ; r)=\left\{y \in \mathbb{R}^{m}:|y-x|<r\right\} .
$$

$$
\begin{aligned}
\mathbb{P}_{x}\left[T_{D}>t\right] \leqslant & \mathbb{P}_{x}\left[\tau_{(\partial D) \cap B(x ; 2 d(x))}>t\right] \\
= & \mathbb{P}_{x}\left[\tau_{(\partial D) \cap B(x ; 2 d(x))}>t, \tau_{(\partial D) \cap B(x ; 2 d(x))}>T_{B^{\circ}(x ; a d(x))}\right] \\
& +\mathbb{P}_{x}\left[\tau_{(\partial D) \cap B(x ; 2 d(x))}>t, \tau_{(\partial D) \cap B(x ; 2 d(x))} \leqslant T_{B^{\circ}(x ; a d(x))}\right] \\
\leqslant & 1-\mathbb{P}_{x}\left[\tau_{(\partial D) \cap B(x ; 2 d(x))} \leqslant T_{B^{\circ}(x ; a d(x))}\right]+\mathbb{P}_{x}\left[T_{B^{\circ}(x ; a d(x))}>t\right] .
\end{aligned}
$$

Let $H$ be an open half space in $\mathbb{R}^{2}$ containing $B^{\circ}(x ; a d(x))$ such that $\partial H$ is tangent to $\partial B^{\circ}(x ; \operatorname{ad}(x))$. Then

$$
\begin{aligned}
\mathbb{P}_{x}\left[T_{B^{\circ}(x ; a d(x))}>t\right] & \leqslant \mathbb{P}_{x}\left[T_{H}>t\right] \\
& =(\pi t)^{-1 / 2} \int_{[0, a d(x))} e^{-q^{2} /(4 t)} d q \leqslant a d(x)(\pi t)^{-1 / 2} .
\end{aligned}
$$

For compact sets $K_{1} \subseteq K_{2}$ we have by the variational formula (2.4)

$$
\operatorname{Cap}\left(K_{1}\right) \leqslant \operatorname{Cap}\left(K_{2}\right) .
$$

Let $x_{0} \in \partial D$ be such that $d(x)=\left|x-x_{0}\right|$. Then $B(x ; 2 d(x)) \supset B\left(x_{0} ; d(x)\right)$, and by (1.3), (2.5) and (2.9)

$$
\begin{aligned}
\operatorname{Cap}((\partial D) \cap B(x ; 2 d(x))) & \geqslant \operatorname{Cap}\left((\partial D) \cap B\left(x_{0} ; d(x)\right)\right) \\
& \geqslant \alpha \operatorname{Cap}\left(B\left(x_{0} ; d(x)\right)\right)=\alpha(d(x))^{1 /(2 \pi)} .
\end{aligned}
$$

By (2.3) and (2.10)

$$
R((\partial D) \cap B(x ; 2 d(x))) \leqslant-(2 \pi)^{-1} \log d(x)-\log \alpha .
$$


By (2.3) and (2.9) we have for $K_{1} \subseteq K_{2}$

Hence

$$
R\left(K_{1}\right) \geqslant R\left(K_{2}\right) .
$$

$$
R((\partial D) \cap B(x ; 2 d(x))) \geqslant R(B(x ; 2 d(x))) \geqslant-(2 \pi)^{-1} \log (2 d(x)) .
$$

Moreover, by (2.1) and (2.2)

$$
\begin{aligned}
\sup \left\{u_{(\partial D) \cap B(x ; 2 d(x))}(y): y \in \partial B(x ; a d(x))\right\} \\
\quad \leqslant-(2 \pi)^{-1} \log ((a-2) d(x)) \int_{(\partial D) \cap B(x ; 2 d(x))} \mu_{(\partial D) \cap B(x ; 2 d(x))}(d y) \\
\quad=-(2 \pi)^{-1} \log ((a-2) d(x)) .
\end{aligned}
$$

Following the proof of [11, Lemma 3.5] we define for $r>0$

and $h: \mathbb{R}^{2} \longrightarrow \mathbb{R}$ by

$$
m(r)=-(2 \pi)^{-1} \log ((a-2) r),
$$

$$
h(y)=(R((\partial D) \cap B(x ; 2 d(x)))-m(d(x)))^{-1}\left(u_{(\partial D) \cap B(x ; 2 d(x))}(y)-m(d(x))\right) .
$$

Now $h$ is superharmonic, harmonic outside $(\partial D) \cap B(x ; 2 d(x))$, equal to one on $(\partial D) \cap B(x ; 2 d(x))$, and by (2.14) negative on $\partial B(x ; a d(x))$. Hence

$$
\mathbb{P}_{x}\left[\tau_{(\partial D) \cap B(x ; 2 d(x))} \leqslant T_{B^{\circ}(x ; a d(x))}\right] \geqslant h(x)=\frac{u_{(\partial D) \cap B(x ; 2 d(x))}(x)-m(d(x))}{R((\partial D) \cap B(x ; 2 d(x)))-m(d(x))} .
$$

But

$$
u_{(\partial D) \cap B(x ; 2 d(x))}(x) \geqslant-\frac{1}{2 \pi} \log (2 d(x)),
$$

so that by (2.11), (2.15), (2.17) and (2.18)

$$
\mathbb{P}_{x}\left[\tau_{(\partial D) \cap B(x ; 2 d(x))} \leqslant T_{B^{\circ}(x ; a d(x))}\right] \geqslant \frac{\log (a-2)-\log 2}{\log (a-2)-\log \alpha^{2 \pi}} .
$$

Hence by (2.7), (2.8) and (2.19)

$$
\mathbb{P}_{x}\left[T_{D}>\lambda_{j}^{-1}\right] \leqslant \frac{\log \left(2 / \alpha^{2 \pi}\right)}{\log \left((a-2) / \alpha^{2 \pi}\right)}+\pi^{-1 / 2} a d(x) \lambda_{j}^{1 / 2} .
$$

We make the following choice for $a$ :

$$
a-2=\frac{2}{\lambda_{j}^{1 / 2} d(x)}\left(1+\log \left(\frac{1}{\lambda_{j}^{1 / 2} d(x)}\right)\right)^{-1} .
$$

Let $z=\lambda_{j}^{-1 / 2} d(x)^{-1}$. Then $d(x) \leqslant \lambda_{j}^{-1 / 2}$ implies $z \geqslant 1$, and $z \geqslant 1+\log (z)$ implies $a \geqslant 4$. By (2.20) and (2.21)

$$
\begin{aligned}
\mathbb{P}_{x}\left[T_{D}>\lambda_{j}^{-1}\right] \leqslant & \left(\log \frac{2}{\alpha^{2 \pi}}\right)\left(\log \frac{1}{\alpha^{2 \pi}}+\log (2 z)-\log (1+\log z)\right)^{-1} \\
& +2 \pi^{-1 / 2} z^{-1}+2 \pi^{-1 / 2}(1+\log z)^{-1}
\end{aligned}
$$

Lemma 2.1. For $z \geqslant 1$

$$
\log (2 z)-\log (1+\log z) \geqslant \frac{1}{2} \log z
$$


Proof. Inequality (2.23) is equivalent to

$$
4 z \geqslant(1+\log z)^{2} \text {. }
$$

But (2.24) holds for $z=1$. Moreover for $z \geqslant 1$

$$
4 \geqslant 2(1+\log z) / z \text {. }
$$

Integration of (2.25) over $[1, z]$ yields $(2.24)$ and hence (2.23).

For $z \geqslant 1,1 / z \leqslant(\log 1 / z)^{-1}$. Hence by Lemma 2.1 and (2.22)

$$
\begin{aligned}
\mathbb{P}_{x}\left[T_{D}>\lambda_{j}^{-1}\right] & \leqslant\left(2 \log \left(\frac{2}{\alpha^{2 \pi}}\right)+\frac{4}{\pi^{1 / 2}}\right)(\log z)^{-1} \\
& \leqslant 6\left(\log \left(\frac{2}{\alpha^{2 \pi}}\right)\right)(\log z)^{-1} .
\end{aligned}
$$

Theorem 1.2 follows from (1.26), (2.26) and by the definition of $z$.

\section{Proof of Theorem 1.3}

We define for $m=3,4, \ldots$ the Green function on $\mathbb{R}^{m}$ by

$$
g(x, y)=\frac{1}{c(m)}|x-y|^{2-m},
$$

where

$$
c(m)=4 \pi^{m / 2}(\Gamma((m-2) / 2))^{-1} .
$$

For a compact set $K \subset \mathbb{R}^{m}$ the equilibrium measure $\mu_{K}$ is the unique non-negative measure on $K$ satisfying

$$
\mathbb{P}_{x}\left[\tau_{K}<\infty\right]=\int g(x, y) \mu_{K}(d y) .
$$

The newtonian capacity of $K$ is defined by

The capacity of a ball is

$$
\operatorname{Cap}(K)=\mu_{K}(K) \text {. }
$$

$$
\operatorname{Cap}(B(0 ; r))=c(m) r^{m-2} .
$$

Again, there is a variational description

$$
\operatorname{Cap}(K)=\left\{\inf _{\mu \in P(K)} \iint g(x, y) \mu(d x) \mu(d y)\right\}^{-1},
$$

where $P(K)$ is the set of all probability measures supported by $K$. If $K_{1}, K_{2}$ are compact sets with $K_{1} \subseteq K_{2}$ then $\operatorname{Cap}\left(K_{1}\right) \leqslant \operatorname{Cap}\left(K_{2}\right)$. For these facts, see for example [18, Chapter 3].

To prove Theorem 1.3 we adapt [10, Lemma 3.5]. Let $b>a>1$. Then by the strong Markov property

$$
\begin{aligned}
\mathbb{P}_{x}\left[T_{D}>t\right] \leqslant & 1-\mathbb{P}_{x}\left[\tau_{(\partial D) \cap B(x ; a d(x))} \leqslant t\right] \\
\leqslant & 1-\mathbb{P}_{x}\left[\tau_{(\partial D) \cap B(x ; a d(x))} \leqslant T_{B^{\circ}(x ; b d(x))}\right]+\mathbb{P}_{x}\left[T_{B^{\circ}(x ; b d(x))}>t\right] \\
\leqslant & 1-\mathbb{P}_{x}\left[\tau_{(\partial D) \cap B(x ; a d(x))}<\infty\right] \\
& +\mathbb{E}_{x}\left[\mathbb{P}_{B\left(T_{B^{\circ}(x ; b d(x))}\right.}\left[\tau_{(\partial D) \cap B(x ; a d(x))}<\infty\right]\right]+\mathbb{P}_{x}\left[T_{B^{\circ}(x ; b d(x))}>t\right] .
\end{aligned}
$$


To estimate the third term in the right-hand side of (3.7) we let $y$ be such that $|y-x|=b d(x)$. Then

$$
\mathbb{P}_{y}\left[\tau_{(\partial D) \cap B(x ; a d(x))}\right] \leqslant \mathbb{P}_{y}\left[\tau_{B(x ; a d(x))}<\infty\right]=\left(\frac{a}{b}\right)^{m-2} .
$$

The fourth term in (3.7) is again estimated by (2.8). Hence

$$
\mathbb{P}_{x}\left[T_{D}>t\right] \leqslant 1-\mathbb{P}_{x}\left[\tau_{(\partial D) \cap B(x ; a d(x))}<\infty\right]+\left(\frac{a}{b}\right)^{m-2}+b d(x)(\pi t)^{-1 / 2} .
$$

Choose $a$ and $b$ as follows:

$$
\begin{gathered}
a=A d(x)^{-\beta_{1}}, \\
b=B(d(x))^{\beta_{2}-1},
\end{gathered}
$$

where $\beta_{1}, \beta_{2}, A$ and $B$ are the solutions of

$$
\begin{gathered}
\beta_{1} \gamma=\left(1-\beta_{1}-\beta_{2}\right)(m-2)=\beta_{2}, \\
A^{-\gamma}=\left(\frac{A}{B}\right)^{m-2}=B \lambda_{j}^{1 / 2} .
\end{gathered}
$$

From (3.12) we obtain

$$
\beta_{2}=\beta_{1} \gamma=\left(\frac{1}{\gamma}+\frac{m-1}{m-2}\right)^{-1}
$$

and from (3.13) we obtain

$$
B \lambda_{j}^{1 / 2}=A^{-\gamma}=\lambda_{j}^{(1 / 2)((1 / \gamma)+(m-1) /(m-2))^{-1}} .
$$

If we can show that (1.7) implies (1.27) and the requirement $b \geqslant a$, then by (3.9)-(3.11) and Theorem 1.7

$$
\begin{aligned}
\mathbb{P}_{x}\left[T_{D}>\lambda_{j}^{-1}\right] & \leqslant 2 A^{-\gamma} d(x)^{\beta_{1} \gamma}+\left(\frac{A}{B}\right)^{m-2} d(x)^{\left(1-\beta_{1}-\beta_{2}\right)(m-2)} \\
& +B d(x)^{\beta_{2}} \lambda_{j}^{1 / 2} .
\end{aligned}
$$

Substitution of the values of $\beta_{1}, \beta_{2}, A$ and $B$ respectively in (3.16) gives

$$
\mathbb{P}_{x}\left[T_{D}>\lambda_{j}^{-1}\right] \leqslant 4 A^{-\gamma} d(x)^{\beta_{1} \gamma}=4\left(d(x) \lambda_{j}^{1 / 2}\right)^{((1 / \gamma)+(m-1) /(m-2))^{-1}} .
$$

Estimate (1.8) follows from (1.26) and (3.17).

Note that $b \geqslant a$ is (by (3.10), (3.11)) equivalent to showing that

$$
\frac{B}{A} \geqslant d(x)^{1-\beta_{1}-\beta_{2}} .
$$

It follows from (3.14) that

$$
0<\beta_{1}+\beta_{2}=\frac{1+\gamma}{1+\gamma \frac{m-1}{m-2}}<1 .
$$

Since (1.7) implies $d(x) \leqslant \lambda_{j}^{-1 / 2}$ it is (by (3.19)) sufficient to check that (3.18) holds for $d(x)=\lambda_{j}^{-1 / 2}$, that is,

$$
\frac{B}{A} \geqslant \lambda_{j}^{-(1 / 2)\left(1-\beta_{1}-\beta_{2}\right)}
$$


We see by (3.15) and (3.19) that (3.20) holds with the equality sign.

It remains to check the validity of (1.27). Since $D$ is bounded, $D$ is contained in a hypercube of sidelength diam $(D)$. By monotonicity of the Dirichlet eigenvalues $[\mathbf{1 9}$, Chapter XIII.15, Proposition 4(a)]

$$
\lambda_{j} \geqslant \lambda_{1}=\frac{m \pi^{2}}{(\operatorname{diam}(D))^{2}}>\frac{1}{(\operatorname{diam}(D))^{2}} .
$$

Hence the first inequality in (1.27) is satisfied if

By (3.10), (3.14) and (3.15)

$$
\frac{1}{\lambda_{j}^{1 / 2} d(x)} \geqslant a
$$

$$
a=\left(\lambda_{j}^{1 / 2} d(x)\right)^{-(1 / \gamma)((1 / \gamma)+(m-1) /(m-2))^{-1}} .
$$

Hence (3.22) is satisfied if

$$
\left(d(x) \lambda_{j}^{1 / 2}\right)^{1-(1 / \gamma)((1 / \gamma)+(m-1) /(m-2))^{-1}} \leqslant 1 .
$$

This is indeed the case because (1.7) implies $d(x) \lambda_{j}^{1 / 2} \leqslant 1$ and

$$
1-\frac{1}{\gamma}\left(\frac{1}{\gamma}+\frac{m-1}{m-2}\right)^{-1}>0 \text {. }
$$

The second inequality in (1.27) follows directly from (3.23) and (1.7).

\section{Proof of Theorem 1.7}

For $s>r>0$ we define the annulus

and the set

$$
B(x ; r, s)=B(x ; s) \backslash B^{\circ}(x ; r),
$$

$$
\partial D_{i}(x)=(\partial D) \cap B\left(x ; b^{i}, b^{i+1}\right),
$$

where $b>1$ will be specified later. Let $A_{i}(x)$ be the event

Define

$$
A_{i}(x)=\left\{\tau_{\partial D_{i}(x)}<\infty\right\} .
$$

$$
N=\max \left\{k \in \mathbb{Z}: b^{k+1} \leqslant a(d(x)) d(x)\right\} .
$$

Then for any $n \leqslant N$

$$
\left\{\tau_{(\partial D) \cap B(x ; a(d(x)) d(x))}<\infty\right\} \supset \bigcup_{i=n}^{N} A_{i}(x) .
$$

If $b^{i+1}<d(x)$ then $A_{i}(x)=\varnothing$. We will choose

$$
n=\min \left\{k \in \mathbb{Z}: b^{k} \geqslant 2 d(x)\right\} .
$$

We choose a 'spacing' $s \in \mathbb{Z}^{+}, s \geqslant 2$ (to be specified later) and use

to obtain

$$
\bigcup_{i=n}^{N} A_{i}(x) \supset \bigcup_{j=0}^{[(N-n) / s]} A_{n+j s}(x),
$$

$$
\mathbb{P}_{x}\left[\tau_{(\partial D) \cap B(x ; \alpha(d(x)) d(x))}<\infty\right] \geqslant 1-\mathbb{P}_{x}\left[\bigcap_{j=0}^{[(N-n) / s]} A_{n+j s}^{c}(x)\right]
$$


For technical reasons we replace $A_{j}(x)$ by $\bar{A}_{j}(x)$ which are defined by

Note that

$$
\bar{A}_{j}(x)=\left\{\tau_{\partial D_{j}(x)} \leqslant \tau_{B\left(x ; b^{j+s}, \infty\right)}\right\} .
$$

and therefore

$$
\mathbb{P}_{x}\left[\bar{A}_{j}(x) \backslash A_{j}(x)\right]=0,
$$

$$
\mathbb{P}_{x}\left[\tau_{(\partial D) \cap B(x ; a(d(x)) d(x))}<\infty\right] \geqslant 1-\mathbb{P}_{x}\left[\bigcap_{j=0}^{[(N-n) / s]} \bar{A}_{n+j s}^{c}(x)\right] .
$$

Next we derive a lower bound for $\mathbb{P}_{y}\left(\bar{A}_{j}(x)\right)$ for $|x-y| \leqslant b^{j}$.

LEMMA 4.1. Let

$$
b=2\left(\frac{2}{\alpha}\right)^{1 /(m-2)} .
$$

Then for $j \geqslant n$ satisfying $b\left(b^{j}+d(x)\right) \leqslant 2 \operatorname{diam}(D)$ and any $y$ satisfying $|y-x| \leqslant b^{j}$

$$
\mathbb{P}_{y}\left[\bar{A}_{j}(x)\right] \geqslant 2 \cdot 3^{-m} \alpha \text {. }
$$

Proof. Let $x_{0} \in \partial D$ be such that $d(x)=\left|x-x_{0}\right|$. One easily checks that if $b^{j} \geqslant 2 d(x)$

where

$$
\partial D_{j}(x) \supset(\partial D) \cap B\left(x_{0} ; r, b r / 2\right),
$$

$$
r=b^{j}+d(x) .
$$

From this we obtain, by the monotonicity and subadditivity of the newtonian capacity,

$$
\begin{aligned}
\operatorname{Cap}\left(\partial D_{j}(x)\right) & \geqslant \operatorname{Cap}\left((\partial D) \cap B\left(x_{0} ; r, b r / 2\right)\right) \\
& \geqslant \operatorname{Cap}\left((\partial D) \cap B\left(x_{0} ; b r / 2\right)\right)-\operatorname{Cap}\left(B\left(x_{0} ; r\right)\right) \\
& \geqslant \alpha \operatorname{Cap}\left(B\left(x_{0} ; b r / 2\right)\right)-\operatorname{Cap}\left(B\left(x_{0} ; r\right)\right),
\end{aligned}
$$

since $b r / 2 \leqslant \operatorname{diam}(D)$ by assumption. By the choice of $b$ and by (3.5)

$$
\operatorname{Cap}\left(\partial D_{j}(x)\right) \geqslant \alpha c(m)(b r / 2)^{m-2}-c(m) r^{m-2}=c(m) r^{m-2} \geqslant c(m) b^{j(m-2)} .
$$

For $z \in \partial D_{j}(x)$ and $|y-x| \leqslant b^{j}$ we have since $b \geqslant 2$

$$
|y-z| \leqslant|z-x|+|x-y| \leqslant b^{j+1}+b^{j} \leqslant 3 b^{j+1} / 2 .
$$

Hence by (4.17) and (4.18)

Furthermore

$$
\begin{aligned}
\mathbb{P}_{y}\left[A_{j}(x)\right] & =\int_{\partial D_{j}(x)} \frac{c(m)^{-1}}{|y-z|^{m-2}} \mu_{\partial D_{j}(x)}(d z) \\
& \geqslant c(m)^{-1}(3 / 2)^{2-m} b^{(2-m)(j+1)} \operatorname{Cap}\left(\partial D_{j}(x)\right) \\
& \geqslant c(m)^{-1}(3 / 2)^{2-m} b^{(2-m)(j+1)} c(m) b^{j(m-2)} \\
& =(3 b / 2)^{2-m} .
\end{aligned}
$$

$$
\mathbb{P}_{y}\left[\bar{A}_{j}(x)\right] \geqslant \mathbb{P}_{y}\left[A_{j}(x)\right]-\mathbb{P}_{w}\left[\tau_{B\left(x ; b^{j+1}\right)}<\infty\right],
$$

where $|w-x|=b^{j+s}$. Hence

$$
\mathbb{P}_{y}\left[\bar{A}_{j}(x)\right] \geqslant(3 b / 2)^{2-m}-b^{(m-2)(1-s)} .
$$


From now on we choose $s=3$. Then by (4.21) and (4.12)

$$
\mathbb{P}_{y}\left[\bar{A}_{j}(x)\right] \geqslant 3^{2-m} \frac{\alpha}{2}-\alpha^{2} 2^{2(1-m)} \geqslant 2 \cdot 3^{-m} \alpha
$$

Let

$$
\mathscr{F}_{j} \equiv \sigma\left(B(t): t \leqslant \tau_{B\left(x ; b^{j}, \infty\right)}\right) .
$$

By definition of $\bar{A}_{j}(x)$, we have that $\bar{A}_{j}(x)$ is $\mathscr{F}_{j+3}$-measurable. Let $x$ be such that

$$
N-n \geqslant 3 \text {. }
$$

Then for any $k \in\{1,2, \ldots,[(N-n) / 3]\}$ we have

$$
\begin{aligned}
& \mathbb{P}_{x}\left[\bigcap_{j=0}^{k} \bar{A}_{n+3 j}^{c}(x)\right]=\mathbb{E}_{x}\left[\mathbb{P}_{x}\left[\bar{A}_{n+3 k}^{c}(x) \mid \widetilde{\mathscr{F}}_{n+3 k}\right] ; \bigcap_{j=0}^{k-1} \bar{A}_{n+3 j}^{c}(x)\right]
\end{aligned}
$$

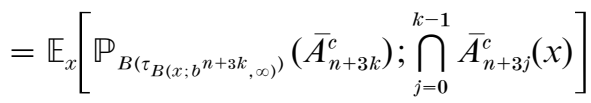

$$
\begin{aligned}
& \leqslant\left(1-2 \cdot 3^{-m} \alpha\right) \mathbb{P}_{x}\left[\bigcap_{j=0}^{k-1} \bar{A}_{n+3 j}^{c}(x)\right] \text {. }
\end{aligned}
$$

From this we finally obtain

$$
\begin{aligned}
\mathbb{P}_{x}\left[\bigcap_{j=0}^{[(N-n) / 3]} \bar{A}_{n+3 j}^{c}(x)\right] & \leqslant\left(1-2 \cdot 3^{-m} \alpha\right)^{[(N-n) / 3]} \\
& \leqslant \exp -\left\{[(N-n) / 3] 2 \cdot 3^{-m} \alpha\right\} .
\end{aligned}
$$

Since $N-n \geqslant 3$,

$$
[(N-n) / 3] \geqslant(N-n) / 6,
$$

and

$$
\mathbb{P}_{x}\left[\bigcap_{j=0}^{[(N-n) / 3]} \bar{A}_{n+3 j}^{c}(x)\right] \leqslant \exp -\left\{(N-n) 3^{-m-1} \alpha\right\} .
$$

By the choice of $N$ and $n$ we have

and hence

$$
\begin{aligned}
b^{N+2} & >a(d(x)) d(x) \geqslant b^{N+1}, \\
b^{n-1} & <2 d(x) \leqslant b^{n},
\end{aligned}
$$

$$
b^{N-n} \geqslant \frac{a(d(x))}{2 b^{3}} .
$$

By (4.28) and (4.31) we obtain

$$
\begin{aligned}
\mathbb{P}_{x}\left[\bigcap_{j=0}^{[(N-n) / 3]} \overline{A_{n+3 j}^{c}}(x)\right] & \leqslant\left(\frac{a(d(x))}{2 b^{3}}\right)^{-\alpha 3^{-m-1} / \log b} \\
& =a(d(x))^{-\gamma} e^{\alpha 3^{-m}+\alpha 3^{-m-1}(\log 2) / \log b} \\
& \leqslant a(d(x))^{-\gamma} e^{4 \times 3^{-m-1}} \leqslant 2 a(d(x))^{-\gamma},
\end{aligned}
$$

by definition of $\gamma$ and the fact that $b \geqslant 2, m \geqslant 3$ and $\alpha<1$. Estimate (1.28) follows from (4.11) and (4.32) provided $x \in D$ is such that (i) $b\left(b^{j}+d(x)\right) \leqslant 2 \operatorname{diam}(D)$ for $j=n, \ldots, N$, (ii) (4.24) holds. But (i) is satisfied if

$$
b^{N+1}+2 b^{N-n+1} d(x) \leqslant 2 \operatorname{diam}(D),
$$

since $b \geqslant 2$. But $b^{N+1} \leqslant a(d(x)) d(x)$ and $2 b^{N-n+1} \leqslant a(d(x))$. So (4.33) and hence (i) are clearly satisfied if

$$
a(d(x)) d(x) \leqslant \operatorname{diam}(D) .
$$


For (4.24) to hold we have to have $b^{N+2-(n-1)} \geqslant b^{6}$. This is the case by (4.29) and (4.30) if

$$
a(d(x)) \geqslant 2 b^{6} .
$$

But $b \leqslant 4 / \alpha$ since $0<\alpha<1$ and $m=3,4, \ldots$. Hence (4.35) and (4.24) hold if

$$
a(d(x)) \geqslant \frac{2^{13}}{\alpha^{6}} .
$$

This completes the proof of Theorem 1.7.

\section{Proof of Theorem 1.5}

Let $G_{D}(\cdot, \cdot)$ be the Green function for $-\Delta_{D}$. Then

$$
G_{D}(x, y)=\int_{0}^{\infty} p_{D}(x, y ; t) d t,
$$

and any Dirichlet eigenfunction of $-\Delta_{D}$ satisfies

$$
\varphi_{j}(x)=\lambda_{j} \int_{D} G_{D}(x, y) \varphi_{j}(y) d y .
$$

By the Cauchy-Schwarz inequality

since $\left\|\varphi_{j}\right\|_{2}=1$.

$$
\left|\varphi_{j}(x)\right| \leqslant \lambda_{j}\left\{\int_{D} G_{D}^{2}(x, y) d y\right\}^{1 / 2}
$$

Lemma 5.1. For $j=1,2, \ldots$

$$
\lambda_{j} \leqslant 8 \pi j R^{-2} .
$$

Proof. By definition of $R, D$ contains an open ball with radius $R$. Hence $D$ contains an open square with sidelength $R \sqrt{2}$. Since the Dirichlet eigenvalues are monotone in $D, \lambda_{j}$ is bounded from above by the $j$ th eigenvalue of this square. The eigenvalues for this square are given by

By definition

$$
\lambda_{k, l}=\pi^{2}\left(k^{2}+l^{2}\right) /\left(2 R^{2}\right), \quad k \in \mathbb{Z}^{+}, l \in \mathbb{Z}^{+} .
$$

$$
j=\#\left\{(k, l): k^{2}+l^{2} \leqslant 2 \lambda_{j} R^{2} / \pi^{2}\right\} .
$$

Suppose $j \geqslant 4$. Then $k^{2}+l^{2} \geqslant 8$ since $j=1$ corresponds to $(k, l)=(1,1)$ and $j=2,3$ corresponds to $(k, l)=(2,1)$ and $(k, l)=(1,2)$. Hence

$$
\lambda_{j} \geqslant \frac{4 \pi^{2}}{R^{2}}, \quad j \geqslant 4 .
$$

But the right-hand side of (5.6) is equal to the number of lattice points in the first quadrant of the disc with radius $R\left(2 \lambda_{j} / \pi^{2}\right)^{1 / 2}$. Hence by (5.6) and (5.7) we have for $j \geqslant 4$

$$
j \geqslant \frac{\pi}{4}\left(\left(2 \lambda_{j} R^{2} / \pi^{2}\right)^{1 / 2}-2^{1 / 2}\right)^{2} \geqslant \frac{\lambda_{j} R^{2}}{8 \pi} .
$$

This proves the lemma for $j \geqslant 4$. The case $j=1,2,3$ is easily verified. 
Let $F$ be the conformal map from the unit disc onto $D$ with $F(0)=x$. Then by the results of $[3, \S 1]$

$$
G_{D}(x, y)=\frac{1}{2 \pi} \log \operatorname{coth}\left(\rho_{D}(x, y)\right),
$$

where

$$
\wp_{D}(x, y)=\inf _{\gamma} \int_{0}^{1} \frac{\left|\gamma^{\prime}(t)\right|}{\left|F^{\prime}(0)\right|} d t,
$$

and where the infimum is taken over all rectifiable curves $\gamma$ in $D$ with $\gamma(0)=x$, $\gamma(1)=y$, and where $F^{\prime}(0)$ is evaluated at $\gamma(t)$. By Koebe's $1 / 4$ theorem

$$
d(\gamma(t)) \leqslant\left|F^{\prime}(0)\right| \leqslant 4 d(\gamma(t)) .
$$

Without loss of generality we may assume that $\gamma$ has a parametrisation with constant speed $c$. Then for any such $\gamma$ we have

By (5.10)-(5.12)

$$
d(\gamma(t)) \leqslant d(x)+t c .
$$

$$
\wp_{D}(x, y) \geqslant \frac{1}{4} \int_{0}^{1} \frac{c}{d(x)+t c} d t=\frac{1}{4} \log \left(1+\frac{c}{d(x)}\right) .
$$

Since $c \geqslant|x-y|$ we have by (5.9) and (5.13)

$$
G_{D}(x, y) \leqslant \frac{1}{2 \pi} \log \frac{(d(x)+|x-y|)^{1 / 2}+d(x)^{1 / 2}}{(d(x)+|x-y|)^{1 / 2}-d(x)^{1 / 2}} .
$$

We note that the right-hand side of (5.14) is positive and strictly decreasing in $|x-y|$ for $x$ fixed. Hence the square of the right-hand side of (5.14) is strictly decreasing in $|x-y|$ for $x$ fixed. Let $R_{0}$ be defined by

$$
\pi R_{0}^{2}=|D| .
$$

By spherical-symmetric rearrangement

$$
\begin{aligned}
\int_{D} G_{D}^{2}(x, y) d y & \leqslant \frac{1}{2 \pi} \int_{0}^{R_{0}} r d r\left(\log \frac{(d(x)+r)^{1 / 2}+d(x)^{1 / 2}}{(d(x)+r)^{1 / 2}-d(x)^{1 / 2}}\right)^{2} \\
& =\frac{2 d(x)^{2}}{\pi} \int_{d(x) / R_{0}}^{\infty} \frac{d r}{r^{3}}\left(\log \left((1+r)^{1 / 2}+r^{1 / 2}\right)\right)^{2} \leqslant \frac{8 d(x) R_{0}}{\pi},
\end{aligned}
$$

since $\log \left((1+r)^{1 / 2}+r^{1 / 2}\right) \leqslant 2 r^{1 / 2}$. The theorem follows from (5.3), (5.4) and (5.16).

COROLlaRy 5.2. Let $D$ be open, simply connected in $\mathbb{R}^{2}$ with finite volume $|D|$. Then

$$
\mathbb{E}_{x}\left[T_{D}\right] \leqslant 2^{3 / 2} \pi^{-3 / 4}|D|^{3 / 4} d(x)^{1 / 2} .
$$

Proof. By the Cauchy-Schwarz inequality

$$
\mathbb{E}_{x}\left[T_{D}\right]=\int_{D} G_{D}(x, y) d y \leqslant|D|^{1 / 2}\left\{\int_{D} G_{D}^{2}(x, y) d y\right\}^{1 / 2}
$$

and (5.17) follows from (5.18), (5.16) and (5.15). 
Acknowledgements. The first author wishes to thank R. Bañuelos for helpful discussions.

\section{References}

1. M. Aizenman and B. Simon, 'Brownian motion and a Harnack inequality for Schrödinger operators', Comm. Pure Appl. Math. 35 (1982) 209-273.

2. R. BAÑUELOS, 'Intrinsic ultracontractivity and eigenfunction estimates for Schrödinger operators', J. Funct. Anal. 100 (1991) 181-206.

3. R. BAÑUELOS, 'Sharp estimates for Dirichlet eigenfunctions in simply connected domains', J. Differential Equations 125 (1996) 282-298.

4. R. BAÑUELOS and M. VAN DEN BERG, 'Dirichlet eigenfunctions for horn-shaped regions and laplacians on cross sections', J. London Math. Soc. 53 (1996) 503-511.

5. R. BAÑUElos and B. DAVIS, 'Heat kernel, eigenfunctions, and conditioned brownian motion in planar domains', J. Funct. Anal. 84 (1989) 188-200.

6. R. BAÑUELOS and B. DAVIS, 'A geometrical characterization of intrinsic ultracontractivity for planar domains with boundaries given by the graphs of functions', Indiana Univ. Math. J. 41 (1992) 885-913.

7. R. BAÑUElos and B. Davis, 'Sharp estimates for Dirichlet eigenfunctions in horn-shaped regions', Comm. Math. Phys. 150 (1992) 209-215.

8. R. BAÑUElos and B. DAvis, correction to 'Sharp estimates for Dirichlet eigenfunctions in hornshaped regions', Comm. Math. Phys. 162 (1994) 215-216.

9. M. VAN DEN BERG, 'Heat content and brownian motion for some regions with a fractal boundary', Probab. Theory Related Fields 100 (1994) 439-456.

10. J. Brossard and R. Carmona, 'Can one hear the dimension of a fractal?', Comm. Math. Phys. 104 (1986) 103-122.

11. E. B. Davies, 'Properties of the Green's function of some Schrödinger operators', J. London Math. Soc. 7 (1973) 483-491.

12. E. B. Davies, 'The equivalence of certain heat kernel and Green function bounds', J. Funct. Anal. 71 (1987) 88-103.

13. E. B. Davies, Heat kernels and spectral theory (Cambridge University Press, Cambridge, 1989).

14. E. B. DAVIES and B. SimON, 'Ultracontractivity and the heat kernel for Schrödinger operators and Dirichlet laplacians', J. Funct. Anal. 59 (1984) 335-395.

15. W. D. Evans, D. J. Harris and R. M. Kauffman, 'Boundary behaviour of Dirichlet eigenfunctions of second order elliptic operators', Math. Z. 204 (1990) 85-115.

16. M. L. LAPIDUS and M. M. H. PANG, 'Eigenfunctions of the Koch snowflake domain', Comm. Math. Phys. 172 (1995) 359-376.

17. Сh. Pommerenke, Boundary behaviour of conformal maps (Springer, Berlin, 1992).

18. S. C. Port and C. J. Stone, Brownian motion and classical potential theory (Academic Press, New York, 1978).

19. M. ReED and B. Simon, Methods of modern mathematical physics. IV: Analysis of operators (Academic Press, New York, 1978).

20. A.-S. Sznitman, Brownian motion, obstacles and random media (Springer, Berlin, 1998).

School of Mathematics

University of Bristol

University Walk

Bristol BS8 1TW

M.vandenBerg@bristol.ac.uk
Institut für Angewandte Mathematik Universität Zürich

Winterthurer Straße 190

CH-8057 Zürich

Switzerland

eb@amath.unizh.ch 